\title{
Alopecia androgénica femenina: nuevos factores fisiopatológicos y futuras tendencias para un abordaje clínico más integral
}

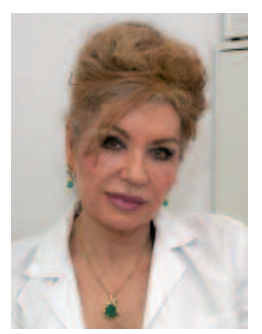

\section{Aurora Guerra Tapia}

Jefa de Sección de Dermatología. Hospital Universitario

12 de Octubre. Madrid.

Profesora titular de Dermatología. Universidad Complutense de Madrid.

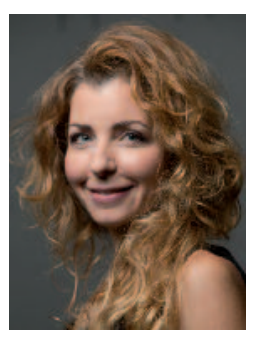

\section{Elena González-Guerra}

Doctora en Medicina y Cirugía.

Médico adjunto

del Servicio de Dermatología.

Hospital Clínico San Carlos. Madrid.

Profesora asociada de Dermatología.

Facultad de Medicina.

Universidad Complutense de

Madrid.

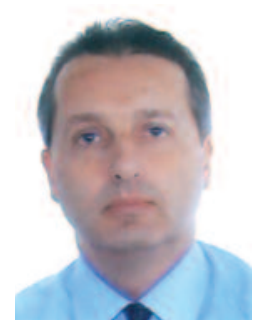

\section{INTRODUCCIÓN}

La alopecia androgénica femenina (AAF) es la principal causa de pérdida de pelo en las mujeres adultas y conlleva un importante impacto psicológico y en la calidad de vida. Se produce por una miniaturización progresiva de los folículos pilosos que conduce hacia una disminución difusa de la densidad del cabello en determinadas áreas del cuero cabelludo, con unas características clínicas y unos patrones histológicos definidos. La patogenia de la AAF responde a un mecanismo fisiopatológico multifactorial, en el que están implicados factores genéticos, hormonales, inflamatorios y oxidativos.

\section{Definición y clínica}

La alopecia androgénica es una forma de alopecia no cicatricial de carácter difuso, producida por una miniaturización progresiva de los folículos del cuero cabelludo, que lleva a una sustitución lenta y progresiva del pelo terminal por cabello velloso. Los ciclos foliculares sucesivos producen cada vez pelos más cortos y de menor diámetro, lo que se traduce en una reducción paulatina del tamaño del cabello hasta volverse inaparente a simple vista en estadios avanzados. Los folículos siguen estando presentes en el cuero cabelludo, pero se encuentran en estado atrófico ${ }^{1,2}$.

En el cuero cabelludo normal de un adulto, aproximadamente un $80-90 \%$ de los pelos están en fase anágena, un 15-20\% en fase telógena y un 1-2\% en fase catágena. En la alopecia androgénica se produce un acortamiento de la fase anágena y una miniaturización de la papila del folículo pilosebáceo, que se manifiestan por un adelgazamiento del cabello. Los cabellos normales se van reemplazando por cabe- 
llos miniaturizados, y con el tiempo dismimuye la densidad capilar en las áreas afectadas. De esta forma, el ciclo anágeno-telógeno se encuentra acortado en el tiempo, y cada vez es mayor la proporción de pelo en fase telógena, que en fase anágena.

Esta forma de alopecia se presenta tanto en mujeres como en hombres; en el caso de las mujeres es más frecuente después de la menopausia. Aunque los cambios foliculares que conducen a la alopecia son similares en hombres y en mujeres, la presentación clínica y la respuesta al tratamiento varían en ambos sexos. Debido a ello, en la actualidad se tiende a denominar a este cuadro clínico en las mujeres como «alopecia de patrón femenino» (female pattern hair loss o FPHL) ${ }^{2}$.

La AAF se caracteriza clínicamente por una pérdida del cabello más difusa que en el hombre, que generalmente mantiene la línea de implantación del pelo. La densidad del cabello se reduce especialmente en el área frontal y superior del cuero cabelludo, aunque con el tiempo puede verse afectada también la región parietal (figs. 1 y 2).

Clínicamente, la AAF se puede manifestar de tres maneras ${ }^{2,3}$ :

1. Pérdida difusa del cabello en ambas regiones biparietales superiores y en la corona, con preservación de la línea de implantación del cabello. Este patrón se clasifica utilizando dos escalas: la de Ludwig, que la divide en tres grados que van desde una pérdida leve de la densidad

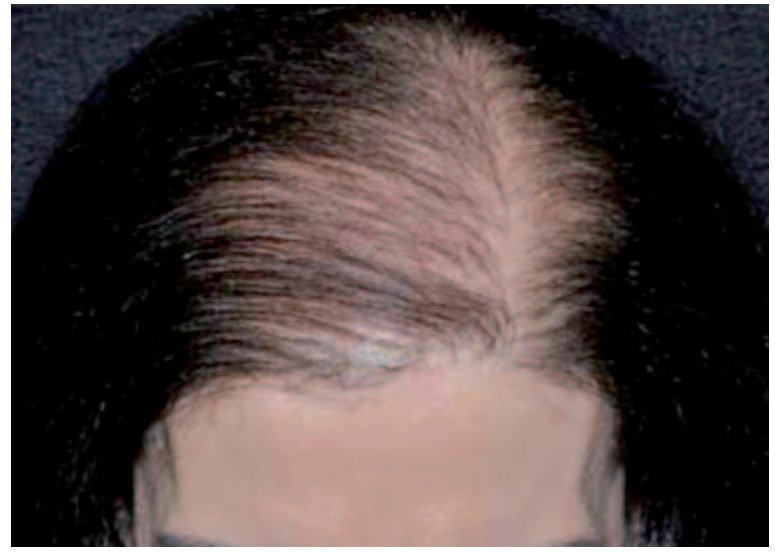

Figura 2. Mujer con alopecia androgénica femenina. Se observa una pérdida difusa del cabello en las áreas frontal y parietal, con la línea de implantación anterior del pelo respetada.

del cabello hasta una completa ausencia de cabellos en la región afectada; y la de Sinclair, que es similar pero con cinco niveles de intensidad.

2. Pérdida difusa del cabello en las regiones bitemporales superiores y en la corona del cuero cabelludo, con acentuación frontal y en la línea central, que se abre en forma de triángulo con su base situada en la línea de implantación anterior del pelo (escala de Olsen: "patrón en árbol de Navidad»).

3. Pérdida difusa del cabello con regresión en la región bitemporal, y existe una recesión de la línea de implantación frontal y alopecia en vértex en distintos grados. Este patrón se utiliza principalmente para la clasificación de la alopecia masculina, pero también puede observarse raramente en las mujeres (patrón de Hamilton).

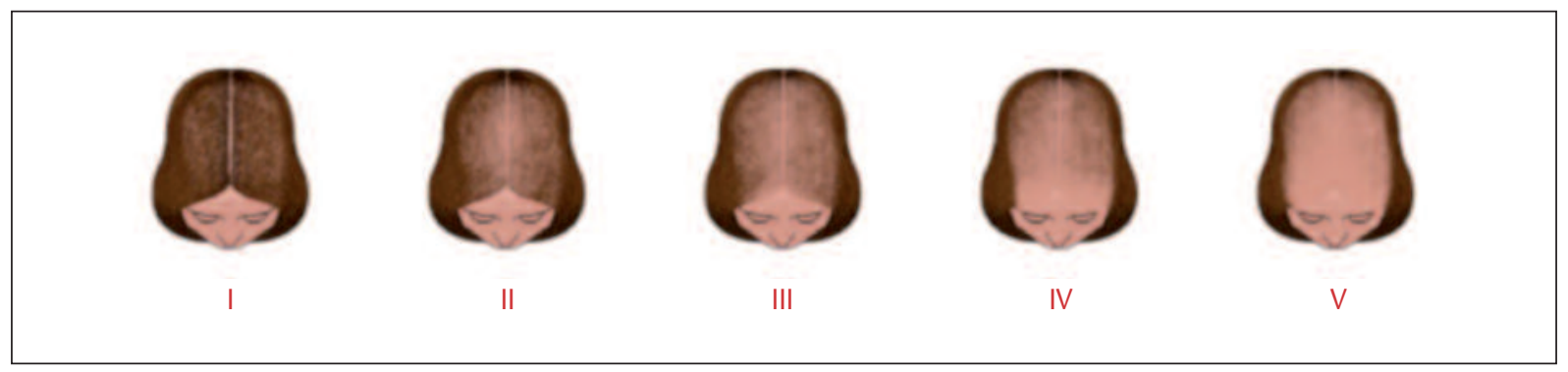

Figura 1. Clasificación de Sinclair. 
Las mujeres suelen ser más conscientes que los hombres de las pérdidas sutiles de cabello y acostumbran a acudir antes al médico. Su característica histopatológica fundamental es un aumento de la proporción de folículos en fase telógena, que empieza a detectarse incluso antes de que la pérdida de cabello sea clínicamente evidente. También se observa una miniaturización de los folículos afectados, que indica que el cabello es más delgado. Cuando la alopecia está bien instaurada, la mayoría de los folículos están acortados y tienen reducido su tamaño ${ }^{4}$.

\section{Epidemiología e impacto}

La frecuencia de la AAF varía entre las distintas poblaciones. Es mucho más común en mujeres de raza caucásica, y menos frecuente en las de etnia asiática y amerindia. Igualmente, la intensidad de esta forma de alopecia varía también dependiendo de la raza: los máximos niveles de intensidad se observan en la población caucásica, los niveles intermedios en las poblaciones asiáticas y afroamericanas, y los menores niveles de intensidad en los esquimales y amerindios ${ }^{5}$.

En la mujer existen dos picos de mayor incidencia a lo largo de su vida, uno en torno a los 30 años de edad y otro hacia los 50-60 años ${ }^{2}$. En términos generales, aproximadamente un $50 \%$ de los hombres y un $40 \%$ de las mujeres padecen alopecia androgénica a los 50 años de edad $(\text { tabla } 1)^{5}$.

Tabla 1. Frecuencia de la alopecia androgénica en mujeres y hombres caucásicos por grupos de edad2,6-8

\begin{tabular}{l|c|c}
\hline Edad & Hombre & Mujer \\
\hline $30-40$ años & $30 \%$ & $15 \%$ \\
\hline $40-50$ años & $40 \%$ & $25 \%$ \\
\hline$>50$ años & $50 \%$ & $40 \%$ \\
\hline$>70$ años & $>80 \%$ & $<50 \%$ \\
\hline
\end{tabular}

La alopecia representa entre el $3 \%$ y el $8 \%$ de las primeras consultas de las clínicas dermatológicas, y de todas las formas de pérdida de pelo, la AAF es la más importante por su frecuencia y repercusión sobre la calidad de vida del paciente.

El cabello es esencial en la identidad de muchas mujeres. La feminidad, la sexualidad, el atractivo y la personalidad están simbólicamente ligadas al cabello femenino, mucho más que en el hombre. La pérdida de pelo en la mujer altera seriamente su autoestima y su autoimagen, y produce un importante impacto negativo sobre la calidad de vida $^{9}$. E1 $52 \%$ de las mujeres están muy preocupadas por la pérdida de pelo, en comparación con el $28 \%$ de los hombres 6 .

Se han diseñado diferentes escalas y cuestionarios para valorar el impacto que la caída del cabello tiene a nivel emocional y en la calidad de la vida de los pacientes afectados. El cuestionario Hairdex $x^{\circledR}$ es una herramienta muy utilizada en este sentido, y consta de 48 preguntas de respuesta múltiple que valoran el estado emocional, la función, los síntomas, la seguridad en uno mismo y la estigmatización. Otra herramienta común es el Symptom Check List-90-R (SCL-90-R), un instrumento de cuantificación sintomática que permite medir la intensidad de 90 síntomas psicológicos y psicopatológicos ${ }^{10}$. Los cuestionarios Dermatology Life Quality Index (DLQI), Short Form-36 Health Survey (SF-36) y Skindex-29 fueron desarrollados para evaluar la calidad de vida en los problemas de la piel, pero también se utilizan en la alopecia ante la escasez de escalas específicas en este campo ${ }^{11}$.

En general, los estudios llevados a cabo utilizando este tipo de escalas en mujeres afectadas por AAF muestran un alto impacto emocional que disminuye seriamente la autoestima y produce un importante impacto negativo sobre la calidad de vida $^{9}$.

\section{FISIOPATOLOGÍA}

Durante años se ha creído que existían solamente dos factores etiopatogénicos en la AAF: el gené- 
tico y el hormonal. Sin embargo, actualmente se considera que existen por lo menos otros dos factores que influyen de manera importante en la aparición de esta forma de alopecia: el estrés oxidativo y la microinflamación.

\section{Factor genético}

Un $50 \%$ de las mujeres con AAF tiene otros familiares afectados, especialmente en las formas de calvicie de inicio precoz, y aunque se acepta que el problema podría transmitirse de forma autosómica dominante con una penetrancia variable, cada vez está más claro que el problema podría seguir un patrón hereditario poligénico ${ }^{2}$.

Las principales evidencias de la participación genética proceden de la secuenciación del gen del receptor para los andrógenos en hombres calvos y no calvos, más conocido como gen $A R$ (androgen receptor), localizado en el cromosoma $\mathrm{X}$ y perteneciente a la familia de factores de transcripción nuclear.

El dominio aminoterminal del gen $A R$ contiene una región codificada por repeticiones de CAG (citosina-adenina-guanina) implicada en los mecanismos de transcripción genética. El número de repeticiones de CAG en la porción aminoterminal varía en cada persona, y se ha observado una relación inversa entre el número de repeticiones y la activación de este gen.

Se ha observado que el riesgo de calvicie es más elevado en aquellas personas con un menor número de repeticiones de $\mathrm{CAG}$, lo que determinaría una mayor actividad del gen $A R$. Este fenómeno se ha observado en la alopecia androgénica masculina y femenina ${ }^{12}$.

\section{Factor hormonal}

Los andrógenos son los principales reguladores del crecimiento del cabello en el humano. Las papilas del folículo pilosebáceo, y en especial las células mesenquimatosas de los folículos pilosos, son objetivos primarios de los andrógenos.

La dependencia de los andrógenos en el desarrollo de alopecia androgénica se conoce desde la antigüedad. En 1942, Hamilton proporcionó la evidencia de que los hombres castrados antes de la pubertad no desarrollaban calvicie, mientras que la administración de testosterona inducía la aparición de calvicie en hombres con antecedentes familiares de alopecia androgénica. Estos factores sugieren la implicación de la testosterona o de alguno de sus metabolitos activos en el desarrollo de alopecia androgénica.

\section{Causas de hiperandrogenismo femenino y perfiles de mujer con alopecia hiperandrogénica}

El término «hiperandrogenismo» se utiliza a menudo para describir los signos cutáneos más comunes en las mujeres con hiperandrogenemia: seborrea, hirsutismo, acné y alopecia. La hiperandrogenemia se acompaña de disfunción ovárica y tiene consecuencias negativas sobre el aparato cardiovascular y el sistema metabólico ${ }^{13}$.

La causa más común de hiperandrogenismo es una excesiva producción androgénica ovárica, suprarrenal o por ambas glándulas. También puede producirse por un comportamiento anómalo de los receptores para los andrógenos en las células diana o por una anomalía en la metabolización periférica de las hormonas esteroideas. Un $15 \%$ de las mujeres en edad reproductiva y sin otros signos de hiperandrogenismo presentan hiperandrogenemia ${ }^{14}$, y La AAF puede ser un signo clínico del hiperandrogenismo.

Dependiendo de los niveles de andrógenos circulantes y del origen del trastorno, la pérdida de cabello puede adoptar diferentes formas. Típicamente, empieza en la corona, pero la alopecia puede adquirir con el tiempo un patrón difuso, con mantenimiento de la línea frontotemporal, o típicamente masculino, con calvicie bitemporal en estados de hiperandrogenemia pronunciada.

La distribución del cabello permite identificar diferentes perfiles de mujer con alopecia androgénica (tabla 2) ${ }^{14}$. 
Tabla 2. Perfiles de mujer con alopecia hiperandrogénica ${ }^{13,14}$

\begin{tabular}{|c|c|c|}
\hline Perfil de alopecia & Imagen & Características de la alopecia \\
\hline AAF Grado I de Ludwig (mínima) & & $\begin{array}{l}\text { Pérdida de cabello en la parte } \\
\text { anterior de la corona, con } \\
\text { ampliación mínima de la raya }\end{array}$ \\
\hline AAF Grado II de Ludwig (moderada) & & $\begin{array}{l}\text { Pérdida de cabello en la parte } \\
\text { anterior de la corona, con } \\
\text { ampliación moderada de la raya }\end{array}$ \\
\hline AAF Grado III de Ludwig (intensa) & & $\begin{array}{c}\text { Corona prácticamente alopécica, } \\
\text { con intensa ampliación de la raya y } \\
\text { conservación de la línea de } \\
\text { implantación anterior del pelo }\end{array}$ \\
\hline $\begin{array}{l}\text { AAF de patrón masculino (AAF.M) } \\
\text { grado I de Ebling }\end{array}$ & & $\begin{array}{l}\text { Alopecia de patrón masculino. Leve } \\
\text { retroceso de la línea de } \\
\text { implantación frontoparietal }\end{array}$ \\
\hline $\begin{array}{l}\text { AAF de patrón masculino (AAF.M) } \\
\text { grado II de Ebling }\end{array}$ & & $\begin{array}{l}\text { Alopecia de patrón masculino. } \\
\text { Retroceso evidente de la línea de } \\
\text { implantación frontoparietal }\end{array}$ \\
\hline $\begin{array}{l}\text { AAF de patrón masculino (AAF.M) } \\
\text { grado III de Ebling }\end{array}$ & & $\begin{array}{l}\text { Alopecia de patrón masculino. } \\
\text { Intenso retroceso de la línea de } \\
\text { implantación frontoparietal }\end{array}$ \\
\hline $\begin{array}{l}\text { AAF de patrón masculino (AAF.M) } \\
\text { grado IV de Ebling }\end{array}$ & & $\begin{array}{l}\text { Alopecia de patrón masculino. Fina } \\
\text { banda de pelo entre la línea de } \\
\text { implantación frontal y la occipital }\end{array}$ \\
\hline $\begin{array}{l}\text { AAF de patrón masculino (AAF.M) } \\
\text { grado } V \text { de Ebling }\end{array}$ & & $\begin{array}{l}\text { Alopecia de patrón masculino. } \\
\text { Depilación frontocoronal total }\end{array}$ \\
\hline
\end{tabular}

Adaptado de Camacho-Martínez ${ }^{14}$.

Mecanismo de activación de la testosterona Los andrógenos circulantes y los andrógenos débiles (deshidroepiandrosterona y androstenodiona), de origen suprarrenal y ovárico en la mujer, se metabolizan en diversos tejidos de la piel. Algunas vías de metabolización, como la conversión de la testosterona en $5 \alpha$-dihidrotestosterona
(DHT), se traducen en un aumento de la potencia androgénica, mientras que en otras vías metabólicas se forman andrógenos más débiles, como los androstenodioles, que participan en las vías de excreción, o los esteroides (fig. 3).

En la AAF existen concentraciones elevadas de $5 \alpha$-reductasa y DHT en los folículos pilosos del 


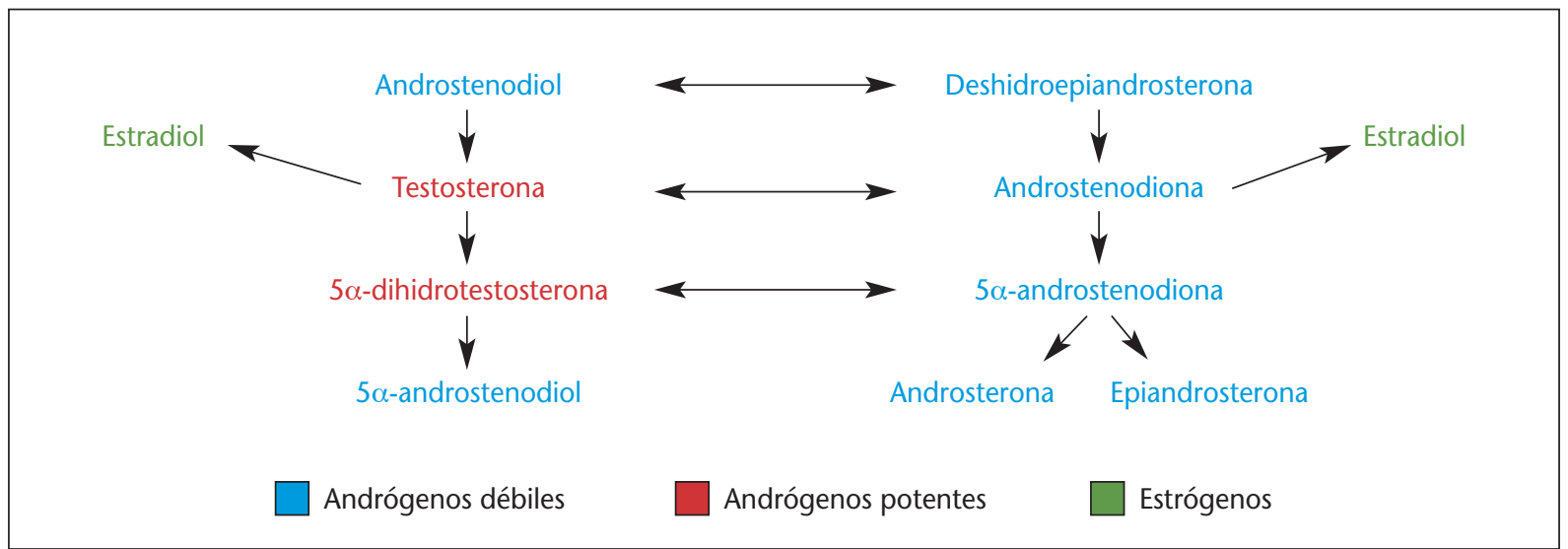

Figura 3. Metabolismo de los andrógenos.

cuero cabelludo. En el interior de las papilas, la testosterona circulante se convierte en DHT por la acción de la enzima $5 \alpha$-reductasa de tipo 2 , codificada por genes ubicados en el cromosoma 2. La DHT es un metabolito activo de la testosterona con una afinidad por los receptores androgénicos cinco veces mayor que la testosterona. La unión de la DHT a su receptor androgénico conduce a la transcripción de los genes responsables de sus acciones biológicas sobre las células diana².

\section{Localización de los receptores para la testosterona en el cuero cabelludo}

Existen dos isoformas de la enzima $5 \alpha$-reductasa: los tipos 1 y 2. En la piel, la $5 \alpha$-reductasa de tipo 1 se expresa en las glándulas sebáceas y en los folículos pilosos, mientras que la de tipo 2 se expresa en las papilas del folículo pilosebáceo y en las células fusiformes interfoliculares de la dermis. Por órganos y sistemas, la $5 \alpha$-reductasa de tipo 1 predomina en el hígado, la piel y el cuero cabelludo, y la de tipo 2 se encuentra más en la piel del tórax y la barba y en los órganos genitourinarios masculinos ${ }^{8,15,16}$.

La distribución de los receptores para la testosterona en el cuero cabelludo, así como la de las enzimas $5 \alpha$-reductasa de los tipos 1 y 2 y el citocromo P-450 aromatasa, que convierte los andrógenos en estrógenos, es distinta en la mujer y en el hombre y varía también en las distintas áreas del cuero cabelludo.

En la mujer y en el hombre, la concentración de receptores para los andrógenos es 1,5 veces mayor en los folículos pilosos frontales que en los occipitales, aunque en la mujer, la concentración de receptores androgénicos en los folículos del área frontal es un $40 \%$ menor que en el hombre. La cantidad de $5 \alpha$-reductasa en el cuero cabelludo también varía en función del sexo y de la presencia de alopecia androgénica. En las mujeres con AAF, la concentración de esta enzima es un $40 \%$ más elevada en la región frontal comparado con los folículos del área occipital, pero su nivel de $5 \alpha$-reductasa es un $50 \%$ inferior al encontrado en los hombres con alopecia androgénica ${ }^{16}$.

En el cuero cabelludo de hombres y mujeres con alopecia androgénica se observan también diferencias importantes en la determinación de la enzima citocromo P-450 aromatasa, con concentraciones un $80 \%$ más elevadas en los folículos del área frontal en la mujer respecto al hombre, que presenta unas concentraciones mínimas de la enzima.

\section{Efectos de la testosterona} sobre el folículo piloso: apoptosis

Dependiendo de la localización de los folículos, los efectos de los andrógenos varían. En la barba, 
las axilas y la región púbica estimulan el crecimiento del pelo, y lo inhiben en la región frontal del cuero cabelludo de individuos genéticamente predispuestos.

Las papilas del folículo pilosebáceo desempeñan un importante papel regulador sobre los folículos pilosos. En el cuero cabelludo, la testosterona circulante penetra en la papila del folículo pilosebáceo (fig. 4), donde puede convertirse en DHT por la acción de la $5 \alpha$-reductasa o permanecer en forma de testosterona. La conversión en DHT dependerá de la ubicación de los folículos; en el cuero cabelludo y en la barba, la testosterona se metaboliza a DHT, mientras que en los folículos del pubis y axilares dicha conversión no se produce.

Para que la DHT actúe debe acoplarse a su receptor androgénico específico. El complejo DHT + receptor se une al ADN y se forma un ARN mensajero que abandonará el núcleo y sintetizará en el citoplasma unas proteínas específicas que inducirán la aparición de determinados efectos en las células y tejidos del folículo (fig. 5) ${ }^{4}$. Las células y tejidos diana incluyen la papila del folículo pilosebáceo, los queratinocitos, los melanocitos, las fibras de tejido conectivo y los pequeños vasos papilares.

En el cuero cabelludo, la DHT induce un aumento de la apoptosis de las células papilares que se produce al final de la fase anágena y se propaga durante la fase catágena. La terminación prematura de la fase anágena es un aspecto clave en la AAF.

En el cabello normal existe un equilibrio entre la producción de factores que promueven la fase anágena y los que promueven la apoptosis. Como posible mecanismo de esta apoptosis aumentada se ha propuesto una mayor producción de factores que promueven la apoptosis folicular, como el factor de necrosis tumoral (TNF)- $\alpha$, la inter-

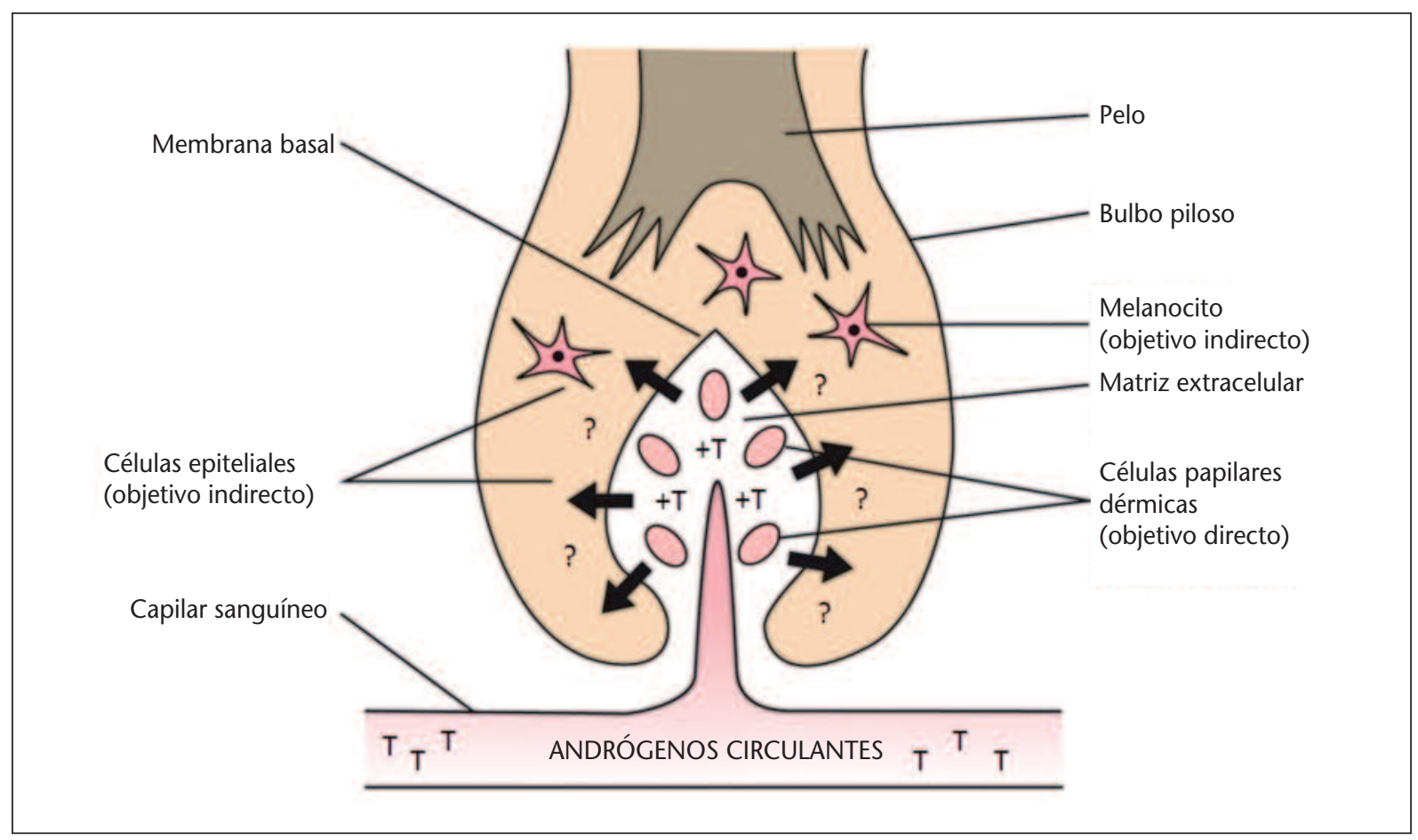

Figura 4. Acción de los andrógenos sobre los folículos del cuero cabelludo.

Adaptado de Randall 4 .

$\mathrm{T}=$ testosterona. 


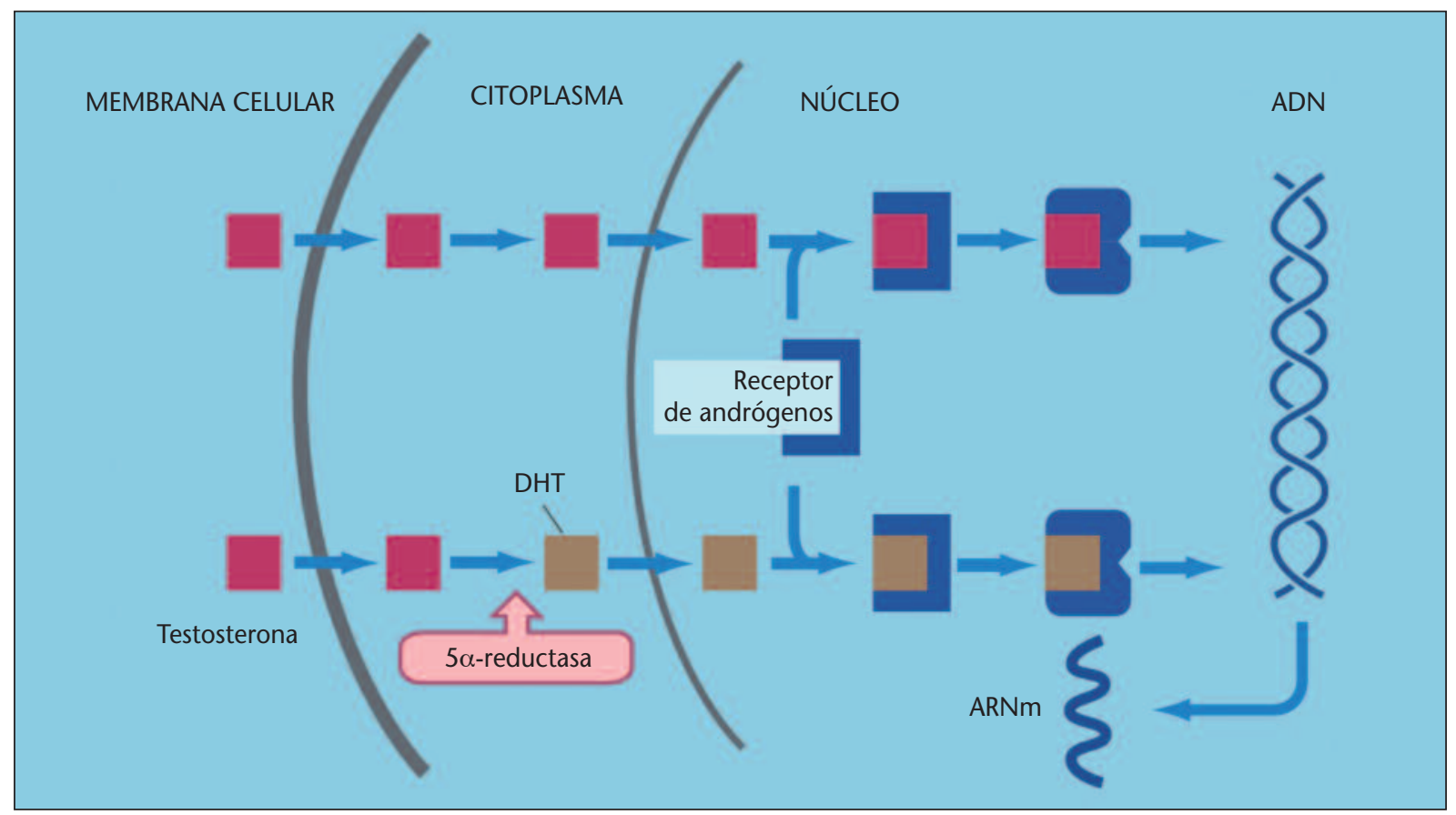

Figura 5. Acción de la dihidrotestosterona en las células foliculares del cuero cabelludo.

Adaptado de Randall4.

leucina (IL)-1 $\alpha$ o el factor de crecimiento transformante (TGF)- $\beta 1^{2}$. En este sentido, se ha observado in vitro que los andrógenos estimulan la producción de TGF- $\beta 1$ (potente inhibidor del crecimiento del cabello) en muestras de folículos pilosebáceos de cuero cabelludo frontal ${ }^{15}$.

\section{Efectos de la testosterona sobre el folículo piloso: incremento de la producción de sebo}

La producción de sebo está muy regulada por la acción de los andrógenos. Los receptores androgénicos también están presentes en los sebocitos, por lo que la DHT actuaría también sobre ellos aumentando su tamaño y la producción de sebo. La asociación de alopecia androgénica y alguno de estos trastornos cutáneos (seborrea, acné, hirsutismo) sugiere un estado de hiperandrogenismo ${ }^{17}$.

Los humanos con síndrome de insensibilidad a los andrógenos no producen sebo, pero cuando existe deficiencia de la enzima $5 \alpha$-reductasa, la producción de sebo es normal. Esto indica que el requisito para la producción de sebo son los andrógenos, pero no necesariamente la DHT.

\section{Efectos de la testosterona sobre el folículo piloso: reducción del crecimiento de los queratinocitos y de la producción de queratina} En el cuero cabelludo, los andrógenos inhiben el crecimiento de los queratinocitos, y como consecuencia se reduce también la producción de queratina. Este efecto podría estar mediado por la liberación de TGF- $\beta 1$ y se ha observado en el cuero cabelludo de hombres con alopecia androgénica, pero no en otras regiones, como la barba, las axilas o el pubis, donde los andrógenos poseen un efecto opuesto al potenciar el desarrollo de los queratinocitos ${ }^{18}$.

Estas diferencias se atribuyen a la distinta expresión de los genes que codifican la producción de 
queratina en las diferentes zonas. Por ejemplo, el gen $h \mathrm{Ha} 7$ para la queratina se detecta en las regiones con vello sexual, pero solamente está presente en determinadas zonas del cuero cabelludo ${ }^{19}$.

\section{Efectos de la testosterona sobre el} folículo piloso: reducción de la circulación Prácticamente todos los pacientes con alopecia androgénica presentan alteraciones en la morfología capilar, con la presencia de múltiples formas tortuosas, dilataciones vasculares y neoformaciones capilares en la piel del cuero cabelludo. El origen de este fenómeno en la alopecia androgénica, que se asocia a una reducción de la microcirculación local, no está claro. Se ha propuesto que podría ser debido a una deficitaria producción de factor de crecimiento endotelial vascular (VEGF), que posee la doble función de aumentar la microvasculatura que rodea el folículo y promover la fase de crecimiento activo del cabello ${ }^{20}$.

\section{Factor oxidativo}

Cada vez surgen más evidencias de que el estrés oxidativo desempeña un papel muy importante en la aparición de la AAF. Los radicales libres procedentes del metabolismo oxidativo, de la exposición a los rayos ultravioleta y de los contaminantes afectan a los folículos pilosos incluso antes del nacimiento del cabello y alteran su posterior crecimiento.

La peroxidación lipídica provocada por los radicales libres induce apoptosis de las células del folículo piloso y acorta la fase anágena, acelerando el paso del cabello a la fase catágena. El proceso de lipoperoxidación consiste en una degradación oxidativa de los lípidos que se inicia por un radical libre que captura electrones de los lípidos en las membranas celulares. En la mayoría de los casos, afecta a los ácidos grasos poliinsaturados, que son «atacados» por especies reactivas de oxígeno (ROS, reactive oxygen species) produciéndose un ácido graso radical que a su vez reacciona con oxígeno, dando lugar a un ácido graso peroxil radical. Este ácido graso peroxil radical es muy inestable y reacciona con otro ácido graso, y se produce un ácido graso radical distinto.

Este ciclo continúa indefinidamente, y se comporta como un mecanismo de reacción en cadena que solo se detendrá cuando dos radicales reaccionan y producen una especie no radical, lo cual es muy improbable. Sin embargo, el organismo humano dispone de enzimas antioxidantes (glutatión, peroxidasas, catalasa, superóxido dismutasa) que atrapan radicales libres e impiden la perpetuación de este proceso, protegiendo así la membrana celular.

Se ha constatado que existe una correlación directa entre el estrés oxidativo, cuya principal manifestación es la producción de ROS, y la aparición de la AAF. Las enzimas antioxidantes los neutralizan e impiden la apoptosis de las células del folículo y el acortamiento de la fase anágena.

Experimentalmente se ha observado que existe un aumento del estrés oxidativo en las papilas del folículo pilosebáceo del cuero cabelludo de hombres con alopecia androgénica. Este estrés oxidativo promueve la liberación de TGF- $\beta 1$, un potente inhibidor del crecimiento del cabello, y se ha vinculado a un aumento de la apoptosis de las células de las papilas del folículo pilosebáceo en las zonas alopécicas y a una menor capacidad de proliferación de estas células. En las papilas del folículo pilosebáceo de las zonas alopécicas del cuero cabelludo existen unas mayores concentraciones de catalasa y glutatión, pero su capacidad para afrontar el estrés oxidativo está reducida en relación con las papilas de la región occipital ${ }^{21,22}$.

\section{Factor inflamatorio}

También en los últimos años surgen más evidencias de que la microinflamación desempeña un importante papel en la aparición de la AAF. Los radicales libres procedentes del metabolismo oxidativo y los procedentes de la inflamación, provocada por una colonización microbiana o diversos irritantes, afectan a los folículos pilosos, como en el caso del daño oxidativo, incluso antes del nacimiento del cabello. 
Se ha confirmado la presencia de fenómenos de microinflamación en las zonas del cuero cabelludo con alopecia androgénica, con infiltración de linfocitos T activados y macrófagos en el tercio superior del folículo piloso, cerca del infundíbulo ${ }^{23}$. Esta localización superior del infiltrado inflamatorio sugiere que el problema causal podría afectar a esta región y permite distinguir esta forma de alopecia de la alopecia areata, en la que también se observa infiltrado inflamatorio, pero localizado en el bulbo y la papila del folículo pilosebáceo.

En un tercio de los casos de alopecia androgénica, los linfocitos T infiltrados, junto con los mastocitos y los macrófagos, perpetúan un estado inflamatorio local. Esta fase de inflamación produce un proceso de remodelado tisular, con la aparición de fibrosis y bandas de colágeno anómalo alrededor de los folículos, en el que pueden desempeñar un papel activo las colagenasas, como las metaloproteinasas de la matriz (MMP)-9, activadas mediante transcripción por citocinas proin- flamatorias, o las MMP-8, producidas directamente por las células infiltrantes (fig. 6).

Se sospecha, por lo tanto, que las colagenasas contribuyen a originar cambios tisulares y a producir fibrosis perifolicular en la alopecia androgénica, que daría lugar a un agrandamiento de la glándula sebácea, un engrosamiento de la vaina dérmica y un depósito anómalo de fibras de colágeno en la parte inferior del folículo piloso ${ }^{24}$. La presencia de fenómenos de microinflamación en el tercio superior del folículo y de remodelación tisular por el desarrollo de fibrosis podría contribuir al proceso de miniaturización del cabello y a la apoptosis aumentada que se observa en la alopecia androgénica, aunque todavía no se ha establecido la relación directa de estos factores.

La observación del infiltrado perifolicular en la parte alta del folículo, cerca del infundíbulo, sugiere una reacción inflamatoria a toxinas o antígenos procedentes de la colonización bacteriana. Los queratinocitos responden al estrés oxidativo y a los irritantes liberando IL-1 $\alpha$ almacenada,

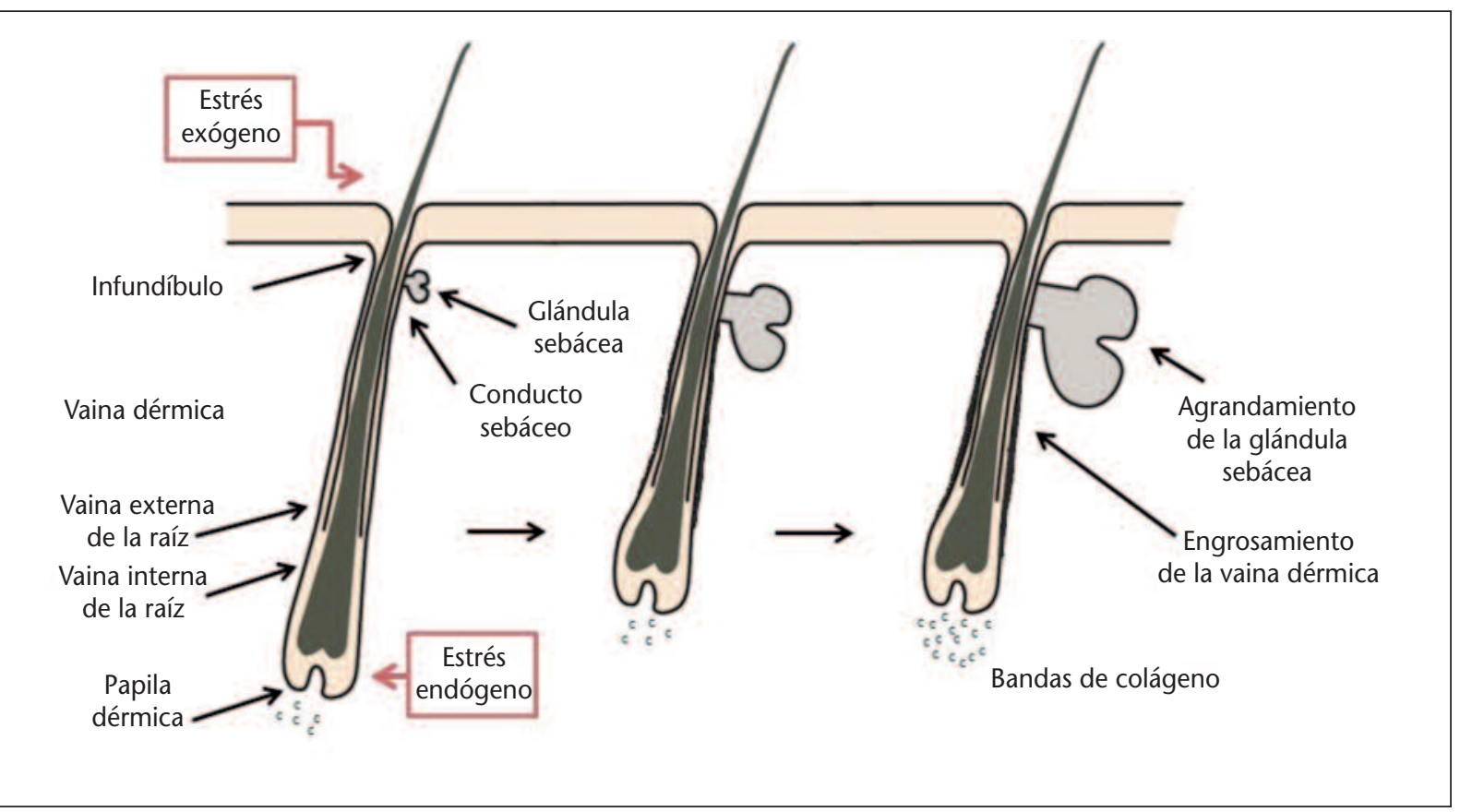

Figura 6. Microinflamación y fibrosis perifolicular en la alopecia androgénica.

Adaptado de Mahé24. 
una citocina proinflamatoria con capacidad para inhibir el crecimiento de folículos ${ }^{24}$. En respuesta a dicha liberación se inicia la transcripción de otros genes sensibles de los queratinocitos adyacentes y se liberan otros intermediarios inflamatorios, como IL- $1 \beta$, TNF- $\alpha$, TGF- $\beta 1$ o IL- 6 , que contribuyen a perpetuar la inflamación.

En conclusión, la fibrosis perifolicular que acompaña a la AAF tiene su origen probablemente en una microinflamación ocasionada por la liberación de citocinas y otros intermediarios inflamatorios que acabarían promoviendo la apoptosis de las células foliculares y, en consecuencia, la reducción de la duración de la fase de anágena.

\section{FUTURAS TENDENCIAS PARA UN ABORDAJE CLÍNICO MÁS INTEGRAL}

La alopecia androgénica femenina tiene muchos puntos que investigar en su etiopatogenia, cuyo conocimiento nos ayudaría a elaborar estrategias que nos permitan un abordaje clínico más integral para su tratamiento. Tal y como hemos desarrollado a lo largo de esta artículo, van surgiendo evidencias sobre el carácter multifactorial de la AAF que exponen el importante papel de nuevos factores, tales como el estrés oxidativo y la microinflamación del folículo piloso. Un análisis en profundidad de estos nuevos factores justificaría nuevas propuestas de tratamiento con una visión terapéutica más amplia, que gracias a un efecto positivo sobre estos nuevos factores mejorarían la eficacia en el tratamiento de la AAF.

Hoy por hoy, parece claro que la terapéutica más eficaz se debe dirigir hacia la inhibición de la $5 \alpha$-reductasa. En este sentido, existen alternativas, entre las que se encuentran aquellas que tienen una mayor actividad inhibitoria de la $5 \alpha$-reductasa y han mostrado ser una opción terapéutica eficaz bien tolerada con mínimos efectos secundarios, como son los extractos lipídicos de Serenoa repens y Pygeum africanum. Sería interesante maximizar tanto como sea posible su actividad inhibitoria de la $5 \alpha$-reductasa, incrementando su efec- tividad inhibitoria y manteniendo su buena tolerabilidad.

La aparición de nuevos factores implicados en la etiopatogenia de la AAF nos lleva a considerar la importancia de incorporar como dianas del tratamiento, ingredientes que además de actuar de una manera sinérgica con la actividad inhibitoria de la $5 \alpha$-reductasa, actúen directamente sobre estos nuevos mecanismos implicados en la patogenia del trastorno, para maximizar la eficacia terapéutica. En este sentido, el factor oxidativo y la microinflamación aparecen, cada vez con más fuerza, como dianas imprescindibles sobre las que se debe intervenir para tratar el proceso.

Otro factor que hay que tener en cuenta es el factor emocional, es decir, el impacto psicológico negativo que puede conllevar la AAF, y que puede afectar tanto a la calidad de vida percibida por la mujer como a su interrelación con el entorno. La AAF es un problema social, que genera un gran sufrimiento en la mujer, mucho más acusado que en el hombre. En este sentido, convendría incorporar a nuestro arsenal terapéutico herramientas que nos permitan una valoración de este factor y de su evolución a lo largo del tratamiento (como pueden ser las escalas de calidad de vida ya mencionadas o nuevas escalas adaptadas y validadas en nuestro entorno), con el fin de detectar estados de salud que requerirán otras estrategias o tratamientos, más allá de aquellos que tienen por objetivos los mecanismos fisiopatológicos de la AAF.

Por otro lado, considerar que el tratamiento de la AAF conlleva un elevado grado de cumplimiento durante largos períodos de tiempo para obtener la eficacia y la efectividad necesaria. Para la mayoría de los autores que han estudiado los factores que influyen en el cumplimiento que la mujer tiene en los tratamientos de larga duración, son tres los principales componentes que hay que tener en cuenta: aceptabilidad, entendida como la percepción que tiene la mujer sobre la seguridad y la eficacia del método, y en general tienen mayor aceptación los tratamientos formulados como suplementos alimenticios (food supplement) que 
los tratamientos hormonales de larga duración; motivación, entendida como actitud positiva de la mujer hacia las características del tratamiento (estar convencida de que el tratamiento es el más adecuado para sus necesidades), y en este sentido es importante la participación informada de la mujer en la elección del tratamiento (con el apoyo del especialista, folletos informativos, programas de formación, etc.), y la continuidad, entendida como la capacidad de usar un tratamiento durante un largo período con el fin de ser constante en el hábito y no tener olvidos o interrupciones, y en este sentido es importante la comodidad en la posología del tratamiento así como su vía de administración y aspecto estético, el incorporar la toma a un hábito cotidiano y el poder contar con un programa de asesoramiento durante el tratamiento que le resuelva posibles incidencias o dudas durante el tratamiento.

Ya para concluir, queremos manifestar que el objetivo de este artículo ha sido realizar una revisión fisiopatológica de la AAF, para obtener una visión global sobre los mecanismos implicados, $\mathrm{y}$ sobre esta visión poder elaborar propuestas futuras para un abordaje clínico integral que mejore la eficacia terapéutica de los tratamientos actuales. Al mismo tiempo, poner de manifiesto que como cualquier otro tratamiento que requiere del cumplimiento, este dependerá de una serie de factores sobre los que podemos influir de manera positiva los diferentes agentes de salud implicados en este abordaje clínico integral de la AAF (médicos especialistas y personal sanitario, farmacéuticos y personal de farmacia, entorno social y familia, industria farmacéutica, etc.).

\section{BIBLIOGRAFÍA}

1. Pierard-Franchimont C, Pierard GE. Alterations in hair follicle dynamics in women. Biomed Res Int. 2013:957432.

2. Müller Ramos P, Amante Miot H. Female pattern hair loss: a clinical and pathophysiological review. An Bras Dermatol. 2015;90(4):529-43

3. Vujovic A, Del Marmol V. The female pattern hair loss: Review of etiopathogenesis and diagnosis. Biomed Res Int. 2014;2014: 767628.

4. Randall VA. Molecular Basis of Androgenetic Alopecia. En: Trüeb RM, Tobin D], editores. Aging Hair. Berlin, Heidelberg: Springer-Verlag; 2010.
5. Thomas J. Androgenetic alopecia: current status. Indian J Dermatol. 2005;50(4):179-90.

6. Dinn QQ, Sinclair R. Female pattern hair loss: current treatment concepts. Clin Interv Aging. 2007;2(2):189-99.

7. Batrinos ML. The endocrinology of baldness. Hormones. 2014;13(2):197-212.

8. Kaliyadan F, Nambiar A, Vijayaraghavan S. Androgenetic alopecia: An update. Indian J Dermatol Venereol Leprol. 2013; 79(5):613-25.

9. Hunt N, McHale $S$. The psychological impact of alopecia. BMJ. 2005;331:951-3.

10. Sawant N, Chikhaltar S, Mehta V, Ravi M, Madke B, Khopkar U. Androgenetic alopecia: Quality-of-life and Associated Lifestyle Patterns. Int J Trichology. 2010;2(2):81-5.

11. Jankovic S, Peric J, Maksimović N, Ćirković A, Marinković J, Janković J, et al. Quality of life in patients with alopecia areata: a hospital-based cross-sectional study. J Eur Acad Dermatol Venereol. 2016;30(5):840-6.

12. Schweiger ES, Boychenko O, Bernstein RM. Update on the pathogenesis, genetics and medical treatment of patterned hair loss. J Drugs Dermatol. 2010;9(11):1412-9.

13. Goodman NF. American Association of Clinical Endocrinologists medical guidelines for clinical practice for the diagnosis and treatment of hyperandrogenic disorders. Endocr Pract. 2001; 7(2):120-34.

14. Camacho-Martinez FM. Hair loss in women. Semin Cutan Med Surg. 2009;28:19-32.

15. Itami S, Inui S. Role of androgen in mesenchimal epithelial interactions in human hair follicle. J Invest Dermatol Symp Proc. 2005;10:209-11.

16. Sawaya ME, Price VH. Different levels of 5alpha-reductase type $\mathrm{i}$ and ii, aromatase, and androgen receptor in hair follicles of women and men with androgenetic alopecia. J Invest Dermatol. 1997; 109:296-300.

17. Degitz KZ. Androgen action on human skin -- from basic research to clinical significance. Exp Dermatol. 2004;13(Suppl. 4):5-10.

18. Cranwell W, Sinclair R. Male androgenetic alopecia. De Groot LJ, BeckPeccoz P, Chrousos G, et al., editores. Endotext [Internet]. South Dartmouth (MA): MDText.com, Inc.; 2000.

19. Jave-Suarez LF, Langbein L, Winter $H$, Praetzel S, Rogers MA, Schweizer $\mathrm{J}$. Androgen regulation of the human hair follicle: The type I hair keratin $\mathrm{hHa} 7$ is a direct target gene in trichocytes. J Invest Dermatol. 2004;122:555-64.

20. Chodorowska G, Michalska-Jakubus M, Bartosińska J, Gerkowicz A, Adamczyk M, Krasowska D. Capillaroscopic patterns in patients with systemic sclerosis, psoriasis and alopecia and their correlations with serum concentrations of several angiogenic markers. Polish J Publ Health. 2015;125(1): 49-54.

21. Upton JH, Hannen RF, Bahta AW, Farjo N, Farjo B, Philpott MP. Oxidative stress-associated senescence in dermal papilla cells of men with androgenetic alopecia. J Invest Dermatol. 2015;135:1244-52.

22. Trüeb RM. Oxidative stress in ageing hair. Int J Trichology. 2009;1(1):6-14.

23. Jaworsky C, Kligman AM, Murphy GF. Characterisation of inflammatory infiltrates in male pattern alopecia: implication for pathogenesis. Br J Dermatol. 1992;127:239-46.

24. Mahé YF, Michelet JF, Billoni N, Jarrousse F, Buan B, Commo $S$, et al. Alopecia androgénica y microinfoamación. Int J Dermatol. 2000;39:576-84. 